INRA Prod. Anim., 2004, 17 (2), 93-108
D. RENAUDEAU ${ }^{1}$, N. MANDONNET ${ }^{1}$, M. TIXIER-BOICHARD ${ }^{2}$, J. NOBLET ${ }^{3}$, J-P. BIDANEL ${ }^{4}$

INRA, Unité de Recherches

Zootechniques, F-97170 Petit Bourg

${ }^{2}$ INRA, Unité Mixte de Recherches Génétique et Diversité Animale, F-78352 Jouy-en-Josas

INRA, Unité Mixte de Recherches sur le Veau et le Porc, F-35590 Saint-Gilles

${ }^{4}$ INRA, Station de Génétique

Quantitative et Appliquée, F-78352 Jouy-en-Josas

Courriel : renaudea@antilles.inra.fr

\section{Atténuer les effets de la chaleur sur les performances des porcs : la voie génétique}

La production mondiale de viande de porc continue d'augmenter, parallèllement à la consommation, et cet accroissement nécessaire concerne surtout des pays en développement. Dans ces pays, les conditions climatiques ne sont pas très favorables à l'élevage des porcs, notament de ceux issus des lignées productives sélectionnées dans les pays tempérés. Plusieurs solutions existent pour atténuer les effets négatifs de la chaleur sur la production ; cet article met en particulier l'accent sur la possibilité de sélectionner des animaux adaptés aux climats chauds.

D'après les projections de la FAO et de l'IRLI (Delgado et al 1999) concernant la production de viande de porc d'ici 2020 , la pro-

\section{Résumé}

La température ambiante est l'un des principaux facteurs climatiques affectant les performances des porcs en période estivale dans les régions tempérées et toute l'année dans les régions tropicales. Les températures ambiantes élevées ont des effets directs principalement sur les fonctions de reproduction du mâle ou de la femelle ou indirectement via une réduction de l'ingestion alimentaire sur les performances de lactation et de croissance. Après avoir brièvement rappelé les principes de la thermorégulation chez le porc et les effets de la chaleur sur leurs performances en fonction du stade physiologique, cet article fait le point sur les solutions utilisables pour atténuer les effets négatifs du climat chaud, en particulier, sur la possibilité de sélectionner des animaux thermotolérants. Cette approche consiste à produire des animaux moins sensibles au stress thermique et/ou ayant une thermorégulation plus efficace. Une des principales difficultés est de comprendre les mécanismes physiologiques impliqués dans l'adaptation à la chaleur et la nature des antagonismes entre les caractères de production et d'adaptation. Cette étape préliminaire conditionne le choix des critères mais également de la meilleure méthode de sélection. Comparativement aux autres espèces (volaille et ruminant), la variabilité génétique de la tolérance à la chaleur et les critères physiologiques ou zootechniques utilisables dans une démarche de sélection chez le porc sont assez peu connus. Cependant, des indicateurs de la sensibilité à la chaleur (Heat Shock Protein), de la thermogenèse (consommation résiduelle, protéines découplantes) ou de la thermolyse (rythme respiratoire, conductivité thermique cutanée) pourraient être des bons critères à considérer pour l'obtention d'une lignée thermotolérante. duction porcine mondiale devrait continuer à se développer principalement dans les pays en voie de développement (Chine, Inde, Asie du Sud-Est, Amérique latine) dans lesquels les conditions climatiques ne sont pas optimales pour l'élevage de porcs. Une des principales conséquences de cette évolution est une intensification des systèmes de production dans ces régions, avec la généralisation de l'importation de lignées commerciales très productives en provenance d'Europe ou d'Amérique du Nord. Dans la plupart des cas, ces lignées ont été sélectionnées dans un environnement optimal permettant de maximiser les performances en minimisant l'effet de l'environnement climatique sur l'expression du potentiel de production des animaux. Dans les régions tempérées, malgré une amélioration des caractéristiques des bâtiments d'élevage (isolation, ventilation), l'augmentation de la température ambiante au cours de certaines périodes de l'année entraîne une perte économique importante pour l'éleveur. Dans un travail de modélisation, St-Pierre et al (2003) estimaient cette perte à environ 310 millions de dollars par an pour la filière porcine américaine.

Lorsque la contrainte climatique est forte, l'optimisation des performances des porcs 
nécessite d'adopter des conduites alimentaires particulières (aliments concentrés en énergie ou à faible extra-chaleur) ou d'investir dans des systèmes de refroidissement coûteux (cooling, brumisation) difficilement transposables économiquement ou techniquement dans la plupart des régions tropicales. Une alternative consisterait à sélectionner des animaux mieux adaptés aux contraintes du climat chaud. Généralement, les races tropicales locales manifestent une bonne adaptation au climat chaud, mais leur niveau de production reste faible. Idéalement, la sélection d'animaux adaptés au chaud ne devrait pas être réalisée au détriment des performances de production. Cette approche nécessite de mettre en ouvre une démarche analytique pour comprendre les mécanismes physiologiques impliqués dans l'adaptation au chaud et les relations entres les caractères d'adaptation et de production. Elle doit également permettre la mise en évidence d'un ou plusieurs critères d'adaptation fiables, faciles à mesurer et indépendants génétiquement des caractères de production. En pratique, la détermination de ces critères est complexe car les mécanismes de l'adaptation à la chaleur font intervenir une multitude de fonctions biologiques. Ceci explique en grande partie le nombre limité de données disponibles dans la bibliographie, notamment chez le porc.

Après un bref rappel des mécanismes de la thermorégulation et des effets de la chaleur sur les performances du porc, cet article présente des solutions pour atténuer ces effets, en mettant l'accent sur la possibilité de sélectionner des animaux adaptés.

\section{1 / Thermorégulation chez le porc}

Le porc est un animal homéotherme capable de maintenir sa température interne constante malgré les variations de la température extérieure. Cette capacité est assurée par la fonction de thermorégulation qui modifie l'équilibre entre la production de chaleur (thermogenèse) et les pertes de chaleurs (thermolyse) pour assurer le maintien de l'homéothermie. Les principes de la thermorégulation chez le porc sont très bien décrits dans la bibliographie (Holmes et Close 1977, Quiniou et al 2000b). L'évolution de la production et des pertes de chaleur en fonction de la température ambiante est illustrée dans la figure 1. La zone de confort thermique est limitée par la température critique inférieure (TCi) et la température critique d'évaporation (TCe) ; elle correspond à la plage de température dans laquelle la dépense énergétique pour la thermorégulation est minimale, constante et indépendante de la température ambiante (Holmes et Close 1977). Ces températures critiques varient en fonction des méthodes et des critères utilisés pour les déterminer (consommation d'aliment, production de chaleur, rythme respiratoire), des conditions d'élevage (composition de l'aliment, humidité et vitesse de l'air ambiant) et, surtout, des caractéristiques de l'animal (stade physiologique, niveau d'alimentation, adiposité ; tableau 1). La TCi est considérée comme un indicateur de la sensibilité à la température ; plus sa valeur est faible et plus l'animal sera sensible à une augmentation de la température ambiante. Dans la zone de confort thermique, des mécanismes peu coû-

Figure 1. Influence de la température ambiante sur la température rectale, la production de chaleur, la consommation d'énergie et les pertes de chaleur par voie latente ou sensible (TCi, TCe et TCs respectivement pour températures critiques inférieure, d'évaporation et supérieure. Adapté de Holmes et Close (1977).

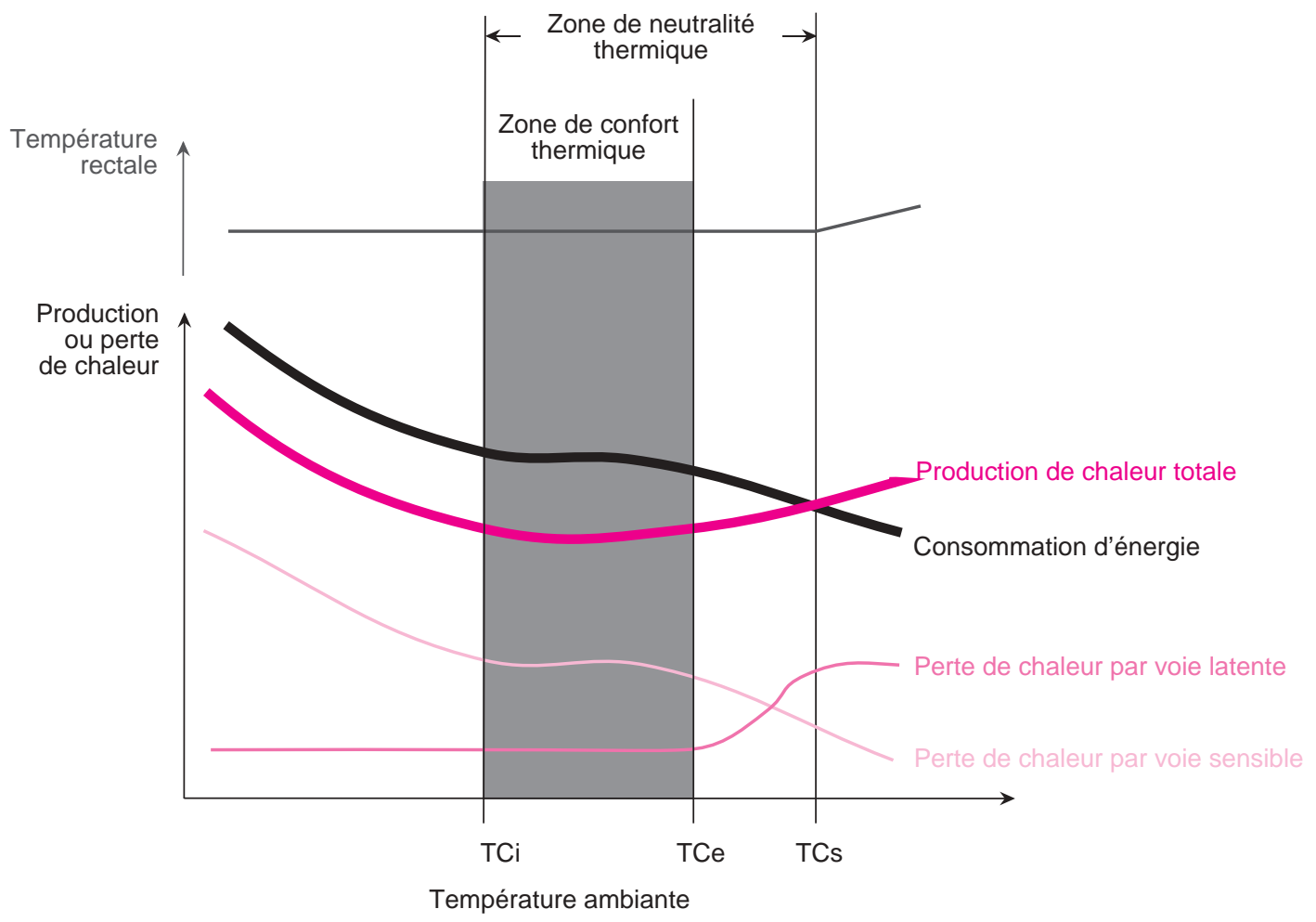


teux (perte de chaleur par la voie sensible et adaptations comportementales) sont mis en place pour maintenir l'homéothermie lorsque la température augmente. Entre la TCe et la température critique supérieure (TCs), le maintien de l'homéothermie passe par une augmentation des pertes de chaleur par évaporation et une diminution de la production de chaleur métabolique. Au-delà de la TCs, les processus impliqués dans la thermorégulation ne suffisent plus et la température interne augmente.

\section{1 / Les pertes de chaleur}

Les échanges entre l'animal et le milieu extérieur s'opèrent suivant deux voies principales : la voie non évaporative (sensible) et la voie évaporative (latente). La perte de chaleur par la voie sensible est basée sur des phénomènes physiques d'échange de chaleur (conduction, convection, radiation) dépendant principalement de la surface d'échange entre l'animal et le milieu extérieur, la conductivité thermique de l'animal (isolation corporelle, pilosité, structure de la peau), du milieu (vitesse de l'air, type de sol) et, enfin, du gradient entre la température à la surface du corps et le milieu ambiant (Ingram 1973). Ces pertes de chaleurs sont peu coûteuses en énergie et peuvent être exprimées grâce à l'équation suivante :

$$
\mathrm{P}=\mathrm{kS}(\mathrm{Tc}-\mathrm{Ta})
$$

où $\mathrm{k}$ est le coefficient de conductance thermique, S la surface d'échange, Tc la température corporelle et $\mathrm{Ta}$ la température ambiante.

Au-delà de la TCi, la température cutanée augmente chez le porc en croissance (Giles et al 1998) et chez la truie en lactation (Quiniou et Noblet 1999). Cette augmentation de la température cutanée résulte d'une redistribution du flux sanguin vers les capillaires sous-cutanés, ce qui augmente la conductance thermique interne et les échanges thermiques entre l'animal et son milieu (Collin et al 2001a). Cependant, le gradient de température entre la peau et l'air ambiant diminue au fur et à mesure que la température ambiante s'accroît, induisant une réduction de l'efficacité des pertes sensibles (Quiniou et al 2000b). Au chaud, la faible pilosité des porcs augmente également la conductance thermique de la peau (Dauncey et Ingram 1986) ; la moindre quantité d'air emprisonnée dans le pelage diminue l'isolation corporelle et augmente les pertes sensibles par convection. Ces échanges de chaleur sont fortement dépendants de la vitesse de l'air autour de l'animal (Ingram 1973). A court terme, la surface d'échange avec l'environnement chez le porc exposé au chaud s'accroît par une modification du comportement social. Le groupe de porcs s'éparpille pour diminuer les contacts entre congénères et maximiser la surface d'échange avec le sol (Ye et Xin 2000). A moyen ou long terme, la morphologie du porc est modifiée. Les animaux sont plus longilignes et la taille des extrémités (oreilles, pattes, queue, groin) augmente, ce qui accroît la surface de déperdition de la chaleur (Dauncey et Ingram 1986). Enfin, une redistribution des masses adipeuses des sites externes (bardière) vers les sites internes (panne) permet d'augmenter la conductance thermique de la peau (Le Dividich et Rinaldo 1989).

Lorsque la température ambiante s'élève au-dessus de la TCe, les pertes sensibles diminuent et les pertes par évaporation devien-

Tableau 1. Variation de la température critique inférieure (TCi) en fonction du stade physiologique, du mode de logement et du niveau d'alimentation (rapporté au besoin d'entretien en énergie métabolisable) chez le porc.

\begin{tabular}{|c|c|c|c|c|}
\hline Stade physiologique & Logement & $\begin{array}{c}\text { Niveau } \\
\text { d'alimentation }\end{array}$ & $\mathrm{TCi}\left({ }^{\circ} \mathrm{C}\right)$ & Référence \\
\hline \multirow[t]{11}{*}{ Truie en gestation } & Individuel & 1,1 & $23-24$ & Kemp et al 1987 \\
\hline & Individuel & 1,3 & $19-20$ & \\
\hline & Individuel & 1,0 & 25 & Holmes et Close 1977 \\
\hline & Individuel & 2,0 & 15 & \\
\hline & Groupe & 1,0 & 14 & Verstegen et Curtis 1988 \\
\hline & Individuel & 1,0 & 20 & Geuyen et al 1984 \\
\hline & Groupe & 1,0 & 14 & \\
\hline & Individuel (maigre) & 1,3 & 18 & Holmes et McLean 1974 \\
\hline & Individuel (grasse) & 1,4 & 11 & \\
\hline & Individuel & 1,2 & 23 & Noblet et al 1989 \\
\hline & Individuel & 1,5 & 20 & \\
\hline \multirow[t]{2}{*}{ Truie en lactation } & Individuel & \# 2 à 3 & 12 & Black et al 1993 \\
\hline & Individuel & \# 2 à 3 & $<18$ & Quiniou et Noblet 1999 \\
\hline \multirow[t]{4}{*}{ Porc en croissance } & Individuel & 2 & 20,5 & Verstegen et al 1978 \\
\hline & Individuel & 3 & 15,5 & \\
\hline & Groupe & 2 & 17 & Holmes et Close 1977 \\
\hline & Groupe & \# 3 & $23-24$ & Quiniou et al 2000a \\
\hline Verrat & Individuel & 1,5 & 20 & Kemp et al 1989 \\
\hline
\end{tabular}


nent plus importantes. L'évaporation d'eau s'effectue par la peau (sudation) et par les poumons (polypnée). Contrairement aux ruminants, les pertes de chaleur évaporatives au niveau des poumons sont prédominantes chez le porc compte tenu de son faible nombre de glandes sudoripares actives (Ingram 1973). Ainsi, lorsque la température ambiante dépasse la température critique d'évaporation (TCe d'environ 25 et $20^{\circ} \mathrm{C}$, respectivement chez le porc en croissance et la truie en lactation), la fréquence des ventilations pulmonaires augmente de façon curvilinéaire en fonction de la température ambiante (Quiniou et al 2000b). L'évaporation de l'eau au niveau de la peau devient un moyen très efficace de dissipation de la chaleur lorsque les porcs ont accès à une source d'humidité (par exemple une mare dans le cas des porcs élevés en plein air).

\section{2 / La production de chaleur}

Les réactions biochimiques n'ont pas un rendement de $100 \%$ et une grande partie de l'énergie ingérée est dissipée sous forme de chaleur. Du point de vue zootechnique, la thermogenèse représente la production de chaleur associée à l'utilisation de l'énergie métabolisable (EM) pour l'entretien et la production (croissance et reproduction). En d'autres termes, elle est égale à la différence entre l'EM ingérée et l'énergie fixée dans les tissus ou exportée dans le lait et peut représenter plus de $50 \%$ de l'énergie ingérée chez le porc en croissance (Van Milgen et al 2000). Elle représente aussi la somme de la production de chaleur liée à l'entretien, à l'activité physique et aux pertes d'énergie pour les productions (croissance, production de lait..). D'un point de vue expérimental, seule la production de chaleur totale est mesurable sur un animal ; il est cependant possible de la décomposer en trois composantes en utilisant des techniques de modélisation (Van Milgen et al 2000, figure 2) : la production de chaleur à jeun, celle liée à l'activité physique et celle associée à l'utilisation métabolique de l'aliment.

Figure 2. Répartition des différentes composantes de la production de chaleur chez le porcelet, le porc en croissance et la truie gestante. D'après Van Milgen et al (2000).

\footnotetext{
HPact : Production de chaleur liée à l'activité physique

TEF : Effet thermique de l'aliment

FHP : Production de chaleur à jeun
}

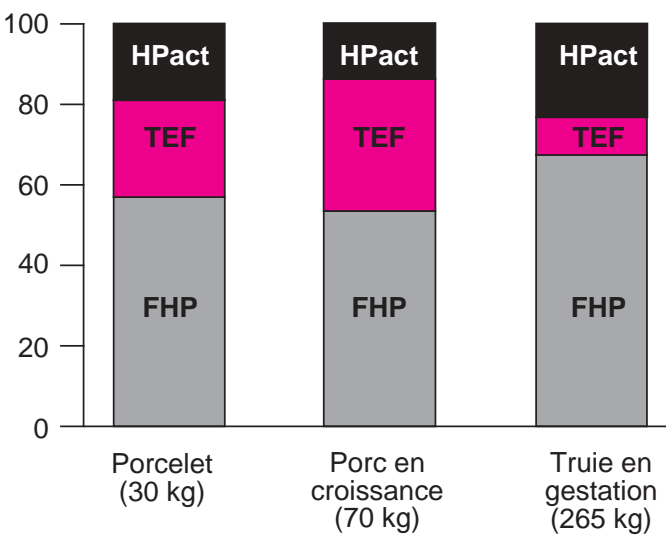

La production de chaleur à jeun (ou FHP pour Fasting Heat Production) est la composante la plus importante de la production de chaleur totale (55 à $70 \%$ selon le stade physiologique). Elle varie principalement en fonction de la durée de la mise à jeun, du poids du tractus digestif, de la composition corporelle (Van Milgen et al 1998) et du niveau alimentaire préalable (De Lange et al 2002). Ces auteurs montrent une réduction de la FHP chez les génotypes gras (Meishan) par rapport aux génotypes maigres (Large White, Piétrain), en partie expliquée par une augmentation du poids des viscères et une réduction de la teneur en muscle. La sélection sur le taux de maigre des carcasses et/ou sur la vitesse de croissance conduit donc à des animaux ayant une production de chaleur basale plus élevée.

La production de chaleur liée à l'activité physique des animaux varie de manière importante en fonction du stade physiologique (environ 15 et $23 \%$ de la production de chaleur totale chez le porc en croissance et la truie gestante) et du mode de logement (en groupe ou individuel) (Noblet et al 1997).

La production de chaleur associée à l'utilisation digestive et métabolique de l'aliment (ou TEF pour Thermic Effect of Feed) représente environ $15 \%$ de l'EM ingérée et varie en fonction du niveau d'ingestion, de la composition nutritionnelle de l'aliment (parois végétales > protéines $>$ amidon $>$ matières grasses) et de l'utilisation finale de ces nutriments (Van Milgen et al 2001). L'efficacité de l'utilisation de l'énergie (rapport énergie nette-EN/EM) est plus importante pour le dépôt de lipides que pour le dépôt de protéines (environ 0,80 vs. 0,60 selon Noblet et al 1999). Par conséquent, pour une même quantité d'énergie déposée, la production de chaleur sera 1,8 fois plus importante si ce dépôt est réalisé sous forme de protéines que de lipides.

En conclusion, les lignées actuellement produites ont une thermogenèse accrue de par l'augmentation de leurs besoins d'entretien et de production, et de par leur capacité plus importante à déposer des protéines. Ces lignées devraient donc être plus sensibles au chaud que les lignées peu ou pas sélectionnées. Ceci semble être confirmé par l'étude de Nienaber et al (1997) montrant une réduction d'environ $4^{\circ} \mathrm{C}$ du seuil à partir duquel les performances de croissance commencent à chuter chez une lignée maigre par rapport à une lignée non sélectionnée.

\section{2 / Effets de la chaleur sur les performances des pores}

\section{1 / La truie en lactation}

A la mise bas, la truie est particulièrement sensible à l'augmentation brusque de la température ambiante. A partir d'une enquête portant sur plus de 30000 truies, D'Allaire et al (1996) rapportent une mortalité des truies cinq à six fois plus importante dès lors que la température de la maternité dépasse $33^{\circ} \mathrm{C}$ au moment de la mise 
bas. Comparée aux autres stades physiologiques, la truie en lactation est particulièrement sensible aux températures ambiantes élevées, en relation avec sa faible TCi (cf. tableau 1). Ceci est en grande partie lié à son niveau d'ingestion élevé pour satisfaire les besoins nutritionnels associés à sa production laitière.

Au-delà de la TCe $\left(20-22^{\circ} \mathrm{C}\right.$ selon Quiniou et al 2000b), la consommation d'aliment chez la truie en lactation diminue de 2,6 MJ ED par jour et par degré (Renaudeau 2001, compilation des résultats de la bibliographie). Mais cette diminution n'est pas linéaire (figure 3). Elle est environ six fois plus importante entre $25^{\circ} \mathrm{C}$ et $29^{\circ} \mathrm{C}$ qu'entre 18 et $25^{\circ} \mathrm{C}$, ce qui tend à montrer qu'au-dessus de $25^{\circ} \mathrm{C}$, la réduction de la consommation d'aliment devient la seule possibilité qu'a l'animal pour maintenir son homéothermie (Quiniou et Noblet 1999). A notre connaissance, peu de résultats sur les effets du climat tropical sont disponibles dans la bibliographie. Renaudeau et al (2003a) montrent un effet important de la saison sur la consommation d'aliment malgré une différence de température moyenne de seulement $2,5^{\circ} \mathrm{C}$ et ces auteurs suggèrent que l'effet de la température élevée est accentué par une forte hygrométrie ambiante.

La réduction de la consommation due à la chaleur accentue le déficit nutritionnel de la truie. En dessous de $25^{\circ} \mathrm{C}$, la mobilisation des réserves corporelles permet de maintenir la croissance des porcelets et la production de lait en compensant la réduction de la consommation. Au-dessus de $25^{\circ} \mathrm{C}$, la mobilisation des réserves n'est plus suffisante pour compenser la baisse de l'appétit et la production laitière et la vitesse de croissance des porcelets diminuent (Quiniou et Noblet 1999). En plus de son effet sur la consommation alimentaire, il semble que la température ambiante ait également un effet direct sur le métabolisme de la glande mammaire via une redistribution du flux sanguin vers la peau au détriment de la mamelle (Black et al 1993). Cependant, des mesures du débit artériel et des prélèvements mammaires montrent que cette vasodilatation sous-cutanée ne se ferait pas au détriment du fonctionnement de la mamelle chez les truies multipares (Renaudeau et al 2003b).

La création de lignées hyperprolifiques dans les races Large White et Landrace puis l'arrivée des lignées sino-européennes ont eu comme conséquence une augmentation de la production laitière et des besoins nutritionnels des truies en lactation. Cet accroissement du potentiel de production s'est accompagné d'une augmentation (moins importante) de la consommation d'aliment et de la production de chaleur métabolique. Nous pouvons donc supposer que l'amélioration de la prolificité des truies s'est traduite par une sélection indirecte d'animaux plus sensibles à la chaleur.

\section{2 / La truie en gestation}

Compte tenu du faible niveau d'alimentation des truies en gestation (rationnées à 1,3 1,5 fois leur besoin d'entretien), l'exposition à la chaleur a peu de conséquence sur leur métabolisme. Elle a en revanche des effets marqués sur la fonction de reproduction. D'importantes variations saisonnières des performances de reproduction (intervalle sevrage-œestrus, taux de conception, mortalité embryonnaire) sont rapportées en climat tempéré (Peltoniemi et al 2000) et tropical (Kabuga et Annor 1991). Ces problèmes peuvent avoir plusieurs origines. Lallongement de l'intervalle entre le sevrage et l'ostrus dépend principalement de l'amplitude de la mobilisation des réserves et donc de l'adéquation entre le niveau d'ingestion et les besoins nutritionnels pour la production laitière. Sur ce point, les truies primipares semblent beaucoup plus sensibles que les truies multipares (Quesnel et Prunier 1995). Cependant, l'augmentation de la durée d'éclairement au cours de la saison estivale est aussi un facteur expliquant les retards dans le retour en ostrus en été (Prunier et al 1996). La réduction de la fécondité et l'augmentation de la mortalité embryonnaire en saison estivale semblent être la conséquence directe d'une forte température dans le mois suivant la saillie (Enne et Greppi 1993), mais également des effets du climat sur les performances du verrat.

\section{3 / Le verrat}

Des travaux menés en milieu tempéré (Guillouet et al 1999) ou tropical (Steinbach 1976) montrent que les performances de reproduction des verrats varient en fonction de la saison. Mais, comme pour la truie gestante, l'effet d'une température élevée sur le métabolisme du verrat est sans doute faible compte tenu de sa température critique inférieure élevée (environ $20^{\circ} \mathrm{C}$, Kemp et al 1989), en relation avec son niveau alimentaire proche de celui de l'entretien. La température aurait un effet direct sur la spermatogenèse via une modification de la synthèse de testostérone (Wettmann et Bazer 1985). Cette altération de la spermatogenèse entraîne une diminution de la mobilité et une augmentation des anomalies morphologiques des spermatozoïdes ; le volume d'éjaculat ne semble pas affecté (Wettmann et al 1979). Par ailleurs, le taux de conception est significativement réduit chez les truies inséminées avec de la semence provenant de verrats préalablement exposés à une température élevée (Wettmann et al 1979). Une partie de la réduction du taux de conception des truies en saison chaude pourrait donc être attribuée à une diminution de la fertilité des verrats.

\section{4 / Le porc en croissance}

Les effets du climat, et en particulier des températures ambiantes élevées, sur les performances du porc en croissance alimenté à volonté sont bien décrits dans la bibliographie (revues de Rinaldo et Le Dividich 1991, Quiniou et al 2000b). Comme pour la truie en lactation, la réduction de l'appétit est une des principales conséquences de l'augmentation de la température. En moyenne, cette réduction de la consommation est de 40 à 80 g/jour par ${ }^{\circ} \mathrm{C}$ pour des températures maintenues 
artificiellement constantes entre 20 et $30^{\circ} \mathrm{C}$ (Collin 2000) et de 16 à $56 \mathrm{~g} /$ jour par ${ }^{\circ} \mathrm{C}$ chez des porcs élevés dans un bâtiment semiouvert en région tropicale (Rinaldo et al 2000). Cette importante variabilité observée entre les études est principalement attribuée à des différences de type génétique, de traitement thermique (gamme de température, constante ou fluctuante, hygrométrie) et de poids vif des animaux. En effet, la chute d'appétit est d'autant plus élevée que les porcs sont lourds (cf. figure 3). La réduction de la vitesse de croissance des porcs observée au chaud est la conséquence directe de la diminution de la quantité de nutriments disponibles pour la croissance (Nienaber et al 1987). Cette étude rapporte également une augmentation du taux de viande maigre dans les carcasses lorsque la température ambiante augmente. Cet effet est directement lié à l'effet de la restriction alimentaire. En revanche, pour un même niveau d'ingestion, Brown-Brandl et al (2000) mesurent une réduction de la vitesse de croissance, du dépôt de protéines et une augmentation du dépôt de lipides au chaud comparativement à la thermoneutralité. Ces résultats, confirmés par Le Bellego et al (2002b), suggèrent que la température ambiante a un effet direct sur les performances de croissance des porcs et sur la répartition de l'énergie ingérée entre le dépôt de protéines et le dépôt de lipides.

Figure 3. Evolution de la prise alimentaire en fonction de la température selon le stade physiologique, à partir des équations proposées par Quiniou et Noblet (1999) pour la truie en lactation, par Quiniou et al (2000a) pour le porc en croissance et finition et par Collin et al (2001b) pour le porcelet sevré. La consommation d'EM est rapportée au besoin d'entretien soit $1 \mathrm{MJ} / \mathrm{kg}^{0,60}$ par jour pour le porc (Noblet et al 1999) et $0,44 \mathrm{MJ} / \mathrm{kg}^{0,75}$ par jour pour la truie (Noblet et Etienne 1987). Adapté de Quiniou et al (2000b).

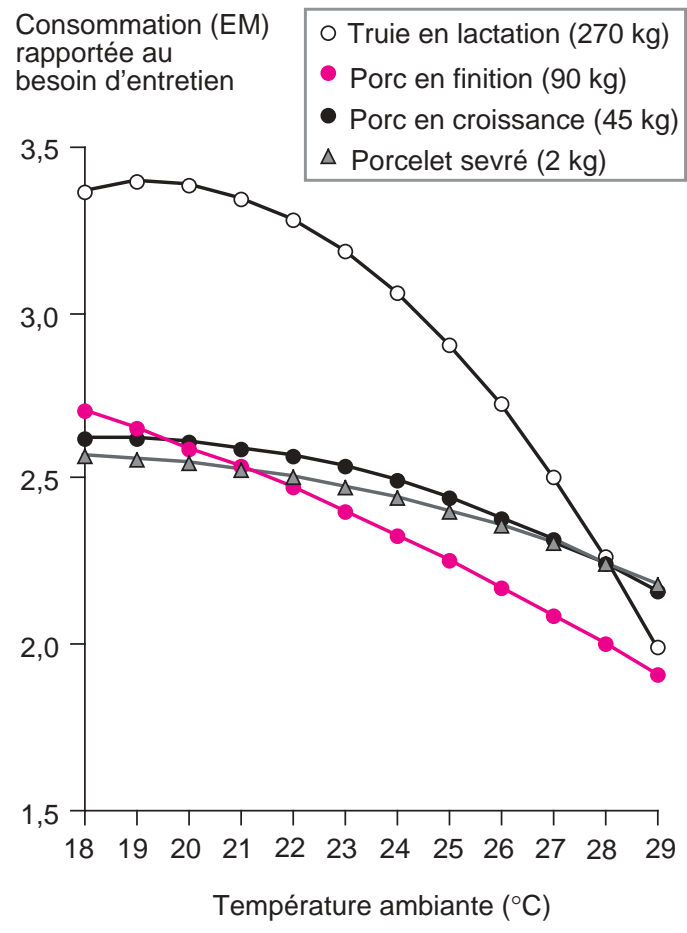

\section{3 / Méthodes permettant d'atténuer les effets de la chaleur}

\section{1 / Modifications de la conduite d'élevage}

Comme nous l'avons vu précédemment, la réduction de l'ingestion d'aliment est une des principales conséquences de l'augmentation de la température ambiante. Pour compenser cette diminution, on peut envisager de concentrer l'aliment et/ou de réduire son extra-chaleur. L'enrichissement de l'aliment en matières grasses présente l'avantage d'atteindre ces deux objectifs. Il a été testé chez le porc en croissance élevé au chaud : l'indice de consommation est amélioré, mais les effets sur la consommation d'énergie et la vitesse de croissance sont négligeables (Stahly et Cromwell 1979, Le Bellego et al 2002a). Dans certains cas, la concentration de l'aliment provoque une augmentation de l'adiposité de la carcasse. De la même manière, l'ajout de matières grasses n'a pas d'effet très marqué sur les performances des truies en lactation élevées au chaud (Schoenherr et al 1989, Renaudeau et al 2001). Lorsqu'il existe, l'effet bénéfique sur la vitesse de croissance de la portée est limité (Quiniou et al 2001). Une réduction de l'extra-chaleur de l'aliment peut être également obtenue en diminuant sa teneur en protéines avec ou sans ajout de matières grasses. Au chaud, l'utilisation de ce type d'aliment a également des effets limités sur les performances de croissance (Le Bellego et al 2002a) et de lactation (Renaudeau et al 2001).

En conclusion, modifier la composition nutritionnelle de l'aliment n'atténue que partiellement la réduction des performances des porcs au chaud et ceci quel que soit le stade physiologique considéré. Par ailleurs, le surcoût induit par l'ajout de matières grasses ou la supplémentation en acides aminés de synthèse dans les régimes à basse teneur en protéines rend difficile l'utilisation de tels aliments dans le contexte tropical où le coût alimentaire est déjà élevé. D'autres techniques, testées chez d'autres espèces (par exemple le poulet) pourraient s'envisager chez le porc. Citons par exemple le fractionnement alimentaire qui consiste à augmenter l'ingestion journalière par des distributions d'aliment au cours des périodes fraîches de la journée.

Des techniques permettant de rafraîchir les animaux ou l'ambiance des bâtiments d'élevage peuvent atténuer les effets de la température. L'utilisation de goutte à goutte sur la tête ou les épaules des animaux augmente les pertes de chaleur par la voie latente. Ce système a été testé avec succès, en particulier chez les truies en lactation (Mc Glone et al 1988). Dans le bâtiment, la diminution de la température ambiante peut être obtenue en utilisant le principe du pouvoir évaporatif de l'air. Les deux équipements les plus utilisés sont la brumisation et les filtres humides ou pad cooling. Bien que relativement coûteux, 
ces dispositifs peuvent permettre de lutter efficacement contre les effets de la chaleur. Ainsi, l'utilisation du pad cooling permet d'augmenter de $25 \%$ la consommation chez des truies en lactation en période estivale au Brésil (Sartor et al 1999). En revanche, de par leur principe de fonctionnement, l'efficacité de tels systèmes est fortement réduite lorsque l'hygrométrie ambiante est élevée ; ceci peut limiter leur utilisation dans les régions tropicales humides. Enfin, d'autres systèmes comme l'augmentation de la vitesse de l'air ou l'utilisation de certains types de caillebotis sont susceptibles d'accroître les pertes de chaleur du porc.

\section{2 / Sélection d'animaux adaptés au climat}

Cette approche consiste à produire des animaux dont les performances ne sont pas ou sont peu réduites par une température élevée. Il s'agit donc de modifier le seuil de sensibilité à la chaleur et/ou d'améliorer l'efficacité de la thermorégulation (i.e. réduction de la thermogenèse et/ou augmentation de la thermolyse). Deux grands types d'adaptation peuvent être considérés : l'adaptation non génétique, ou acclimatation, et l'adaptation génétique.

Lacclimatation représente la capacité de l'animal à ajuster son métabolisme ou son comportement au cours du temps pour mieux tolérer une température élevée (figure 4). Ces ajustements font appel à des réponses coordonnées à différents niveaux d'organisation (structural, organique, cellulaire et moléculaire) et provoquent des modifications de l'expression de certains gènes, d'activités enzymatiques, de taille d'organe, de dépôt de tissus ou de consommation d'énergie (Collier et al 2002). D'après Maloyan et al (1999), l'adaptation à la chaleur est un processus de type biphasique. Dans un premier temps, l'adaptation se caractérise par une stimulation rapide par le système nerveux autonome des effecteurs permettant d'augmenter la dissipation de la chaleur (glandes sudoripares, vaisseaux sous-cutanés...). Dans un second temps, la production de chaleur est réduite en augmentant l'efficacité du fonctionnement des organes et/ou en diminuant la prise ali-

Figure 4. Evolution du niveau de performance d'un animal exposé à un stress thermique de longue durée : un exemple d'acclimatation au chaud.

\section{Niveau de performance}

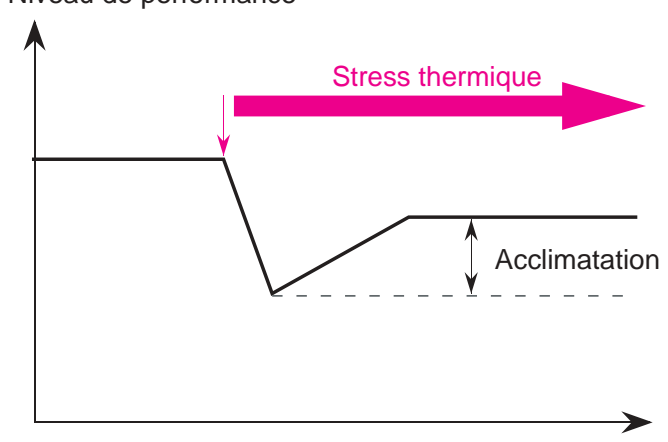

Temps mentaire. Cependant, l'ensemble de ces réponses disparaît avec le stress thermique et n'est pas transmissible à la descendance. Un cas particulier d'adaptation non génétique a également été mis en évidence : une habituation à la chaleur à un certain moment de la vie des animaux peut modifier de manière permanente leur réponse lors d'un nouveau stress thermique. Ainsi, la capacité des poulets de chair à supporter un stress thermique aigu est fortement améliorée lorsque les animaux ont préalablement été exposés à une forte température durant la période néonatale (De Basilio et Picard 2002). Cependant ce type d'adaptation se rapproche de l'acclimatation par le fait qu'il n'est pas transmis à la descendance.

L'expression 'adaptation génétique' est utilisée lorsque les réponses observées précédemment sont fixées dans le patrimoine génétique de l'animal. Elle peut être décom-

Figure 5. Exemple d'adaptation génétique à la chaleur. A : le seuil de sensibilité au chaud est déterminé génétiquement, $B$ : la vitesse d'adaptation au chaud (angle a) est déterminée génétiquement, et $C$ : la diminution des performances au chaud (b) est déterminée génétiquement.

- A -

Niveau de performance $\quad$ Animal 1

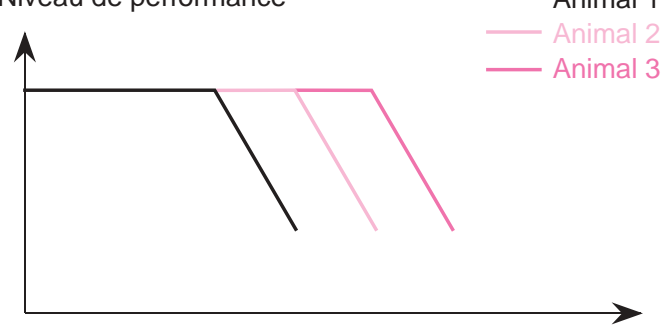

Niveau de stress thermique

- B -

Niveau de performance

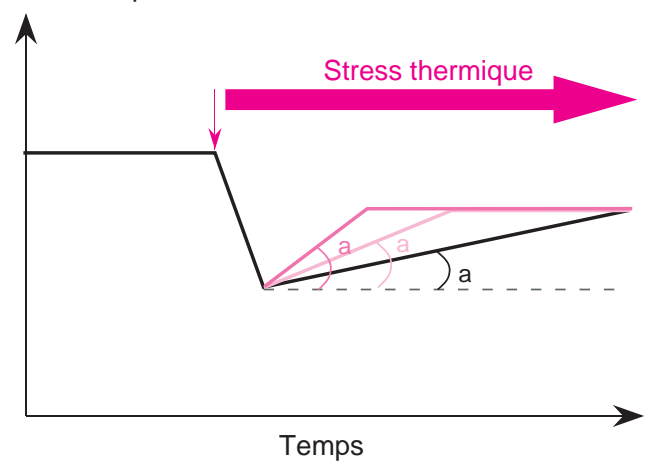

- C -

Niveau de performance

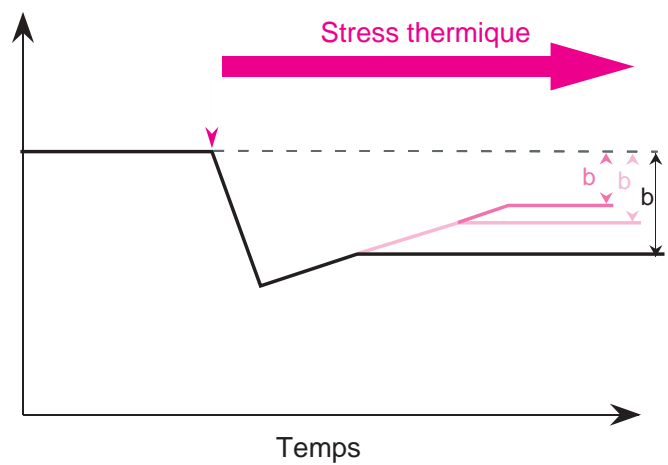


posée en une composante génétique acquise au fil des générations sous l'action de pressions sélectives et une composante épigénétique (plasticité phénotypique). Chez les ruminants, les races spécialisées de type Bos taurus sont beaucoup moins bien adaptées à la chaleur que certaines races locales tropicales de type Bos indicus (Kadzere et al 2002). Dans cet exemple, la faculté de ces animaux à moins souffrir d'un climat chaud est en grande partie due à des particularités physiologiques ou morphologiques acquises sous la pression de la sélection naturelle. L’adaptation génétique peut être déclinée en une augmentation du seuil de sensibilité au chaud ainsi que de la vitesse et de la capacité d'acclimatation à un stress thermique chronique (figure 5).

La plasticité phénotypique représente la capacité d'un génotype ou d'un individu à modifier l'expression d'un ou plusieurs caractères en réponse à un changement de milieu. Elle peut être caractérisée par sa courbe de réponse à l'environnement, encore appelée norme de réaction (figure 6 ). Celle-ci peut également présenter une variabilité génétique pouvant autoriser la sélection d'une norme de réaction optimale. Létude du déterminisme génétique des normes de réaction est donc une approche séduisante dans la problématique de l'adaptation génétique à la chaleur.

\section{a / Variabilité génétique de l'adapta- tion à la chaleur}

La sélection d'animaux adaptés au chaud implique que l'aptitude à tolérer la chaleur ait un fondement génétique. L'existence de différences d'adaptation à la chaleur entre ou intra types génétiques suggère que tel est le cas.

Chez les bovins ou les volailles, une variabilité génétique de l'adaptation au chaud a été clairement mise en évidence entre différents types génétiques. L'aptitude de certains génotypes à mieux tolérer le chaud est d'abord la conséquence d'une mise en place de caractéristiques morpho-physiologiques visant à augmenter leur capacité à dissiper la chaleur. Chez les bovins, ces adaptations consistent en une augmentation de la surface d'échange, de la conductivité thermique de la peau et de la capacité respiratoire et de sudation (Finch 1985). Par ailleurs, une production de chaleur métabolique plus faible de certaines races explique en partie leur bonne adaptation au climat chaud. Celle-ci est dépendante du niveau de production des animaux (Baker et

Figure 6. Normes de réaction pour deux animaux.

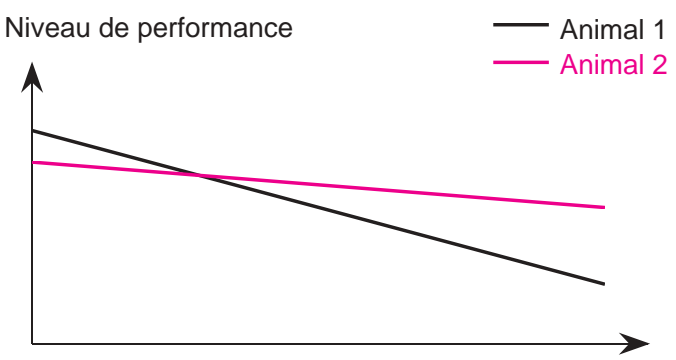

Niveau de stress thermique
Rege 1994, Kadzere et al 2002). Peu de résultats comparables sont disponibles chez le porc. Tauson et al (1998) mettent en évidence une réduction de la capacité à tolérer les fortes températures chez des génotypes sensibles à l'halothane comparés aux génotypes non sensibles. Nienaber et al (1997) montrent une réduction du seuil de sensibilité aux fortes températures chez une lignée de porcs très maigres par rapport à une lignée non sélectionnée.

A l'intérieur d'une population, la variabilité génétique de la tolérance à la chaleur a également été mise en évidence, notamment chez la volaille. Settar et al (1999) observent une importante variabilité génétique pour la tolérance à la chaleur dans une population de poulets de chair qu'ils relient à des différences de potentiel de croissance entre les individus. A notre connaissance, des résultats comparables ne sont pas disponibles chez le porc. Cependant, de nombreux travaux de la bibliographie montrent une forte variabilité individuelle dans la réponse à l'exposition au chaud (Renaudeau 2001).

\section{b / Amélioration génétique de l'adapta- tion à la chaleur}

Plusieurs méthodes peuvent conduire à l'amélioration génétique du niveau de production des animaux lorsqu'ils sont élevés au chaud : le croisement entre des animaux locaux adaptés et exotiques ou la sélection à l'intérieur d'un même génotype.

La première méthode consiste à profiter de la vigueur hybride et de la complémentarité des races pour obtenir une amélioration génétique rapide. Cependant cette technique a pour principal inconvénient de n'être pas permanente. Chez le poulet, le croisement entre une lignée tempérée productive (dwarf) et la Fayoumi (race locale égyptienne) permet d'obtenir une lignée F1 ayant un taux de ponte et une efficacité alimentaire significativement plus élevés au chaud (Abd-el-Gawad et al 1992).

Une deuxième méthode consiste à sélectionner dans une population les individus ayant une bonne aptitude à produire au chaud. Par rapport au croisement, le progrès génétique est plus lent, mais présente l'avantage d'être permanent. Deux options peuvent être choisies pour sélectionner dans un milieu tropical des animaux ayant un bon niveau de production et adaptés au milieu tropical (Baker et Rege 1994). La première consiste à sélectionner indirectement des individus adaptés en utilisant comme critère leur capacité à produire au chaud. Une étude australienne (Frisch 1981) a montré que l'utilisation de la vitesse de croissance comme critère de sélection pour améliorer les performances de génisses élevées sous un climat tropical sec a permis une augmentation concomitante des capacités d'adaptation au chaud par rapport à une lignée non sélectionnée. En revanche, lorsque la comparaison des deux lignées était réalisée dans des conditions proches de la thermoneutralité, les performances de croissance des animaux 
sélectionnés étaient fortement réduites. Ce type de sélection est donc très efficace uniquement si elle est réalisée dans un milieu proche de celui de production. Chez le poulet, l'héritabilité du gain de poids est deux fois moins élevée chez des animaux placés à $32^{\circ} \mathrm{C}$ qu'à $22^{\circ} \mathrm{C}$; cet effet n'est pas mis en évidence pour l'indice de consommation (Beaumont $e t$ al 1998). Ces résultats suggèrent l'importance du choix du critère de sélection (dans ce cas l'indice de consommation plutôt que la vitesse de croissance) pour sélectionner des animaux sur leurs aptitudes à produire au chaud. La seconde option consiste à sélectionner à la fois les capacités d'adaptation et le niveau de production en utilisant un indice de sélection combinant les deux groupes de caractères. Cette seconde option est beaucoup plus complexe que la première car elle nécessite de comprendre les mécanismes biologiques et physiologiques impliqués dans l'adaptation à la chaleur et de quantifier les relations entre les caractères d'adaptation et de production. Cependant, elle permet de mieux diriger le choix des animaux lors de la sélection. Une troisième méthode combinant les deux premières (i.e. croisement et sélection intra race) pourrait également être utilisée pour sélectionner des animaux bien adaptés au chaud. En effet, on peut imaginer une sélection massale sur une lignée synthétique obtenue par croisement.

Le choix de la méthode de sélection est conditionné par la nature de l'antagonisme entre les caractères de production et d'adaptation. Dans le cas où cet antagonisme est lié à un déséquilibre de liaison, on suppose que les allèles favorables des gènes de production sont toujours fortuitement associés aux allèles défavorables des gènes d'adaptation. Dans ce cas, une sélection intra race pourrait être utilisée avec des objectifs de sélection combinés de production et d'adaptation. Si l'antagonisme entre production et adaptation est déterminé par un même gène ou groupe de gènes (effets pléiotropiques défavorables), le mode de sélection peut varier en fonction de la nature du caractère d'adaptation. Si ce caractère permet une réduction de la thermogenèse (généralement très liée aux caractères de production), la sélection conjointe des deux objectifs s'avère difficile, seuls les croisements pourront être utilisés. Dans le cas où le caractère d'adaptation est responsable d'un accroissement des capacités de thermolyse (généralement peu liée aux caractères de production), une sélection uniquement sur les gènes impliqués dans la dissipation de la chaleur pourra être envisagée.

Les récents progrès en biologie moléculaire offrent la possibilité d'identifier des marqueurs moléculaires associés à des locus expliquant une grande part de la variance phénotypique ou génétique d'un caractère quantitatif (QTL) et de les utiliser dans des schémas d'amélioration génétique (en sélection assistée ou en introgression). Dans le cas de la résistance à la chaleur, le phénotype étant difficilement mesurable à grande échelle, l'utilisation des outils moléculaires peut s'avérer très efficace (Bidanel et al 2003). Quelle que soit la méthode de sélection choi- sie, il est cependant nécessaire de disposer d'indicateurs fiables de la tolérance à la chaleur pouvant servir directement comme critère de sélection ou indirectement à la détection de QTL. De plus, ces critères doivent être facilement mesurables, peu coûteux, présenter une variabilité génétique suffisante et être peu ou pas corrélés avec les caractères de production.

\section{c / Critères d'adaptation utilisables dans une démarche de sélection}

Un indicateur de la thermorégulation : la température rectale

La température rectale est l'un des critères les plus fréquemment cités dans la bibliographie pour caractériser la sensibilité des animaux au climat : elle est la résultante de l'ensemble des processus de la thermorégulation et sa mesure a pour principaux avantages d'être simple, peu coûteuse et réalisable chez des animaux jeunes. Malgré la multiplicité de facteurs contribuant à la variabilité de la température rectale, une part non négligeable de cette variabilité est d'origine génétique (héritabilité $h^{2}=0,23$ en moyenne, tableau 2), ce qui permet d'envisager une sélection efficace sur ce critère. En revanche, il existe une liaison moyennement à fortement négative, tant au plan phénotypique que génétique, entre la température rectale et les caractères de production (tableau 3). Cependant, la précision de ces estimations, notamment pour les corrélations génétiques, est généralement faible (erreur standard comprise entre 0,17 et 0,40). Cette mauvaise précision est en partie expliquée par la taille des dispositifs expérimentaux utilisés dans certaines études. De plus, le maintien d'une température interne constante peut être obtenu par plusieurs voies de régulation n'ayant pas les mêmes effets sur les performances ; ceci pourrait également expliquer la mauvaise précision des estimations des corrélations génétiques entre la température interne et les caractères de production (Turner 1984). Pour améliorer le niveau de production des animaux élevés au chaud, ces résultats semblent suggérer qu'il est plus intéressant d'utiliser des indicateurs de la production ou de perte de chaleur comme critère de sélection. Cependant, les travaux disponibles dans la littérature ont été le plus souvent réalisés en utilisant comme critère une mesure ponctuelle de la température rectale ; il est probable que les estimations de l'héritabilité ou des corrélations génétiques et phénotypiques faites à partir de variations journalières ou saisonnières de la température rectale soient différentes.

\section{Indicateurs de la production de chaleur}

Une possibilité de sélection pour améliorer la résistance des animaux à la chaleur réside dans la diminution de la dépense énergétique ou de la production de chaleur. Chez le porc, cette dernière pourrait être obtenue en agissant sur une ou plusieurs de ses trois composantes principales : la production de chaleur à jeun (FHP), la production de chaleur liée à l'activité physique et l'effet thermique de l'aliment. 
Tableau 2. Les principaux caractères de la tolérance à la chaleur et leur variabilité génétique.

\begin{tabular}{|c|c|c|c|c|c|}
\hline $\begin{array}{l}\text { Caractère } \\
\text { Critère de mesure }\end{array}$ & Espèce & $\begin{array}{l}\text { Source de la } \\
\text { variabilité } \\
\text { génétique }\end{array}$ & $\mathrm{N}^{(1)}$ & Héritabilité $\mathrm{h}^{2}$ & Référence \\
\hline \multicolumn{6}{|l|}{ Température interne } \\
\hline \multirow[t]{7}{*}{ Température rectale } & bovine & Intra génotype & 611 & 0,19 & Morris et al 1989 \\
\hline & & & 445 & 0,34 & Turner $1984^{(2)}$ \\
\hline & & & 1700 & 0,25 & Turner $19822^{(2)}$ \\
\hline & & & 1341 & 0,19 & Mackinnon et al $1991^{(3)}$ \\
\hline & & & 2403 & 0,17 & Burrow $2001^{(3)}$ \\
\hline & & & 192 & 0,11 & Da Silva 1973 \\
\hline & volailles & Intra génotype & 161 & 0,37 & Taouis et al 2002 \\
\hline \multicolumn{6}{|l|}{ Production de chaleur } \\
\hline FHP & bovine & & 45 & 0,75 & Hotovy et al 1991 \\
\hline $\mathrm{Km}^{(4)}$ & bovine & & 45 & 0,34 & Hotovy et al 1991 \\
\hline Réduction de la taille & volailles & Gène majeur dw & - & - & Bordas et al $1999^{(5)}$ \\
\hline \multirow[t]{4}{*}{ Consommation résiduelle } & bovine & Intra génotype & 510 & 0,43 & Arthur et al 2001 \\
\hline & porcine & Intra génotype & 26706 & $0,15-0,36$ & Johnson et al 1999 \\
\hline & porcine & Intra génotype & 5248 & 0,25 & Labroue et al 1999 \\
\hline & porcine & Intra génotype & 7562 & 0,34 & Mrode et Kennedy 1993 \\
\hline \multicolumn{6}{|c|}{ Perte de chaleur par évaporation } \\
\hline \multirow[t]{2}{*}{ Rythme respiratoire } & bovine & Intra génotype & 192 & 0,59 & Da Silva 1973 \\
\hline & volailles & Intra génotype & 453 & 0,14 & Obeidah et al 1974 \\
\hline $\begin{array}{l}\text { Diamètre des glandes } \\
\text { sudoripares }\end{array}$ & bovine & Intra génotype & $\mathrm{NC}$ & 0,40 & Jenkinson et al 1975 \\
\hline \multicolumn{6}{|c|}{ Perte de chaleur par conduction ou convection } \\
\hline \multirow[t]{2}{*}{ Longueur des poils } & bovine & Gène majeur & & & Olson et al 2003 \\
\hline & bovine & Intra génotype & 345 & 0,08 & Da Silva et al 1988 \\
\hline Epaisseur de la peau & bovine & Intra génotype & 345 & 0,23 & Da Silva et al 1988 \\
\hline Pigmentation de la peau & bovine & Intra génotype & 345 & 0,30 & Da Silva et al 1988 \\
\hline Perte des plumes du cou & volailles & Gène majeur $\mathrm{Na}$ & - & - & Bordas et al $1999^{(5)}$ \\
\hline $\begin{array}{l}\text { Modification de la structure } \\
\text { des plumes }\end{array}$ & volailles & $\begin{array}{l}\text { Gènes majeurs : } \\
\mathrm{F}, \mathrm{K}, \mathrm{h}\end{array}$ & - & - & Bordas et al $1999^{(5)}$ \\
\hline
\end{tabular}

(1) Nombre d'animaux utilisés pour l'estimation de l'héritabilité.

(2) Log (température rectale - 38).

(3) Log (température rectale - 37).

(4) Rendement de transformation de l'énergie métabolisable (EM) pour l'entretien.

(5) Revue bibliographique.

Tableau 3. Corrélations génétiques et phénotypiques (en italique) entre la température rectale et certains caractères de production chez les bovins.

\begin{tabular}{|c|c|c|c|c|c|c|}
\hline Référence & $\begin{array}{r}\text { Po } \\
\text { d'eng }\end{array}$ & $\begin{array}{l}\text { fin } \\
\text { ement }\end{array}$ & & & Crois & après \\
\hline Morris et al 1989 & $-0,08$ & $-0,27$ & & & & \\
\hline Turner 1984 & $-0,16$ & $-0,86$ & & & & \\
\hline Turner 1982 & & & $-0,17$ & $-0,76$ & & \\
\hline Mackinnon et al 1991 & $-0,11$ & $-0,32$ & & & $-0,07$ & $-0,12$ \\
\hline Burrow 2001 & $-0,12$ & $-0,40$ & 0,02 & $-0,16$ & $-0,08$ & $-0,37$ \\
\hline Da Silva 1973 & & & & & $-0,02$ & $-0,23$ \\
\hline
\end{tabular}

Une grande partie de la variabilité de l'activité physique chez le porc dépend des conditions d'élevage et/ou du stade physiologique. Bien qu'il ait été démontré que l'activité physique soit un caractère héritable chez INRA Productions Animales, Mai 2004 les bovins $\left(\mathrm{h}^{2}=0,10\right.$; Boissy et al 2002), chez le porc, il semble plus efficace de réduire le coût énergétique associé à l'activité physique en modifiant, par exemple, les conditions de logement. 
Chez les bovins, Ferrell et Jenkins (1985) suggèrent que la meilleure adaptation de la vache Zébu par rapport à la Holstein est en grande partie expliquée par une réduction d'environ 20 \% de la FHP chez le Zébu. Chez les bovins, une grande partie de la variabilité de la FHP semble avoir une origine génétique $\left(\mathrm{h}^{2}=0,75\right.$; cf. tableau 2$)$ ce qui permet d'envisager une sélection sur ce critère. Chez le porc, des résultats comparables ne sont pas disponibles ; à poids constant une réduction de la FHP est associée à une réduction de la prise alimentaire et/ou à une augmentation du dépôt de gras (voir paragraphe 2.2). Il apparaît donc relativement difficile de sélectionner des animaux ayant une FHP réduite sans provoquer une réduction des performances ou de la qualité de la carcasse.

Chez la souris à l'entretien, une sélection divergente sur la production de chaleur totale a été menée sur 15 générations. Cette sélection a permis de réduire d'environ $22 \%$ la production de chaleur chez la lignée HP- par rapport au témoin, l'héritabilité réalisée étant de 0,26 (Nielsen et al 1997b). Cette réduction de la production de chaleur est en grande partie liée à la diminution de ses composantes liées à l'utilisation digestive et métabolique de l'aliment (i.e. réduction de la prise alimentaire) et à l'activité physique des animaux HP(Nielsen et al 1997a, Mousel et al 2001). De plus, une augmentation de la teneur en gras chez les souris HP- est également observée (Nielsen et al 1997a), suggérant une modification du métabolisme de ces animaux pour favoriser le dépôt de lipides au détriment du dépôt de protéines (énergétiquement moins efficace). Chez les animaux d'élevage, il est difficile de mesurer sur un grand nombre d'individus la production de chaleur; il est cependant possible de l'estimer indirectement en utilisant la consommation résiduelle comme critère. Celle-ci représente en effet la part de la consommation d'aliment qui n'est pas expliquée par les besoins théoriques d'entretien et de production de l'animal. Une sélection sur une faible consommation résiduelle chez la volaille entraîne une réduction de la production de chaleur de 20 à $30 \%$, associée à une réduction de la production de chaleur liée à l'utilisation de l'aliment et à l'activité physique, sans modification des performances (Gabarrou et al 1998). Comme pour la lignée de souris HP-, les poules pondeuses R-sont significativement plus grasses en raison notamment d'une augmentation de l'expression de gènes codant pour des enzymes lipogéniques (Lagarrigue et al 2000). La variabilité génétique de la consommation résiduelle chez le porc est relativement bien décrite dans la bibliographie. L'héritabilité de ce caractère est moyenne (comprise entre 0,15 et 0,34 ; cf. tableau 2 ) et varie selon les études en fonction du type génétique et de l'âge des animaux, mais également en fonction du modèle utilisé pour le calcul de la consommation résiduelle (Mrode et Kennedy 1993, Labroue et al 1999). De plus, les corrélations génétiques entre la consommation résiduelle et la vitesse de croissance ou l'épaisseur de lard sont positives et varient respectivement de 0,06 à 0,49 et de 0,02 à 0,67 (Mrode et Kennedy 1993, Labroue et al 1999).
A notre connaissance, il n'existe pas actuellement de lignées sélectionnées sur la consommation résiduelle chez le porc; une sélection divergente sur ce critère est en cours à l'INRA (J-P. Bidanel et coll.). La consommation résiduelle représente en moyenne $2-5 \%$ de la consommation totale chez le porc (Labroue $e t$ al 1999), ce qui reste relativement faible par rapport à la volaille (environ 10-15 \%) ; nous pouvons donc supposer un effet moins marqué de la réduction de la consommation résiduelle sur la production de chaleur chez les porcins comparativement à la volaille.

A partir de la lignée de souris HP-, une recherche de QTL a été entreprise. Elle a permis de mettre en évidence des QTL influençant la production de chaleur sur les chromosomes 1, 2, 3 et 7 . Pour ce dernier, les gènes codant pour les protéines découplantes UCP2 et UCP3 ont été localisés dans la région chromosomique du QTL (Moody et al 1999). Les protéines UCP2, exprimée dans la plupart des tissus, et UCP3, exprimée uniquement dans les muscles squelettiques, sont des effecteurs importants du métabolisme énergétique des mitochondries (Ricquier et Bouillaud 2000). Ces protéines découplantes ont donc un effet non négligeable sur la thermogenèse.

Chez la volaille, Raimbault et al (2001) montrent une augmentation significative de l'expression des ARNm des UCP musculaires (UCP2 et UCP3) dans la lignée $\mathrm{R}+$ par rapport à la lignée $\mathrm{R}$-. L'expression de ces gènes a également été mise en évidence chez le porcelet âgé de 3 à 5 jours (Damon et al 2000).

Ainsi, une réduction de la production de chaleur en utilisant comme critères de sélection la consommation résiduelle ou l'expression de protéines découplantes pourrait avoir des conséquences positives sur la capacité des animaux à s'adapter au chaud.

Cette hypothèse a été testée chez la volaille. Bordas et Minvielle (1997) montrent que les effets des températures ambiantes élevées sur les performances sont atténuées pour la lignée $\mathrm{R}+$ de poules pondeuses comparativement à la lignée $\mathrm{R}$-. Ce résultat est lié en grande partie à une augmentation des surfaces de déperdition de la chaleur (tarses, barbillons) chez la lignée $R+$, permettant d'accroître leur capacité de perte de chaleur. De plus, par définition, la sélection d'un animal $\mathrm{R}$ - tend à faire coïncider sa consommation d'aliment avec ses besoins. Nous pouvons donc supposer qu'une réduction de la prise alimentaire au chaud a des répercussions importantes sur ces performances. Au contraire, un même stress thermique affectera d'abord la partie résiduelle de la prise alimentaire de l'animal $\mathrm{R}+$, causant peu ou pas d'effet sur ses performances. Ces résultats méritent d'être vérifiés pour d'autres stades physiologiques et chez d'autres espèces, en particulier chez le porc.

\section{Indicateurs de la thermolyse}

Des indicateurs d'une augmentation de la capacité de déperdition de la chaleur peuvent également être utilisés comme critères de sélection pour améliorer génétiquement la tolérance à la chaleur des animaux. 
Comme nous l'avons décrit précédemment, les pertes de chaleur par la voie sensible (conduction, radiation, convection) dépendent de la surface d'échange et de la conductivité thermique de la peau. La conductance thermique dépend de l'isolation corporelle, mais également de la capacité de l'organisme à rediriger le sang des organes internes vers la périphérie. Chez les ruminants, l'augmentation de la capacité de thermolyse non évaporative observée dans les races locales tropicales résulte d'une amélioration de la conductance thermique de la peau (Finch 1985). Cette augmentation est expliquée par une plus grande aptitude à rediriger le sang vers la peau via des ajustements cardio-vasculaires (Giles et Black 1991), mais également des particularités physiques du pelage de l'animal qui font varier sa capacité à retenir de l'air. Ainsi, la bonne thermotolérance de la vache Senepol est en grande partie liée aux caractéristiques de sa robe (poils courts) qui permettent d'augmenter le transfert de chaleur (Hammond et al 1998). La caractéristique « poil court » semble dépendre d'un seul gène (Olson et al 2003). Chez la volaille, un certain nombre de gènes majeurs permettant de réduire l'emplumement (cou nu) ou de modifier la conformation des plumes (plumage frisé) ont été mis en évidence (Geraert 1995). L'utilisation de ces gènes (seuls ou en association) pour la sélection permet d'améliorer sensiblement les performances des poules pondeuses ou des poulets de chair élevés au chaud (Mérat 1986). Chez le porc, une augmentation de la conductivité thermique de la peau des porcs croisés Large White x Créole par rapport à celle des Large White est rapportée par Berbigier (1975) sur un nombre limité d'animaux.

Chez les porcs, les glandes sudoripares étant peu nombreuses et/ou peu actives, les pertes de chaleur par évaporation s'effectuent principalement au niveau pulmonaire. La sélection sur le rythme des ventilations pulmonaires et/ou une capacité pulmonaire devrait donc logiquement permettre d'améliorer l'adaptation des animaux au chaud. Au contraire, les résultats de la bibliographie, en particulier chez les bovins, montrent que les espèces tropicales ont un rythme de ventilation pulmonaire significativement plus faible que les animaux importés des régions tempérées (Carvalho et al 1995). Pour ces auteurs, cela est principalement lié à un seuil de sensibilité (=TCe) plus élevé chez les races tropicales qui résulte d'une augmentation de la thermolyse sensible et/ou d'une réduction de la production de chaleur métabolique. Une partie non négligeable de la variabilité du rythme des ventilations pulmonaires est d'origine génétique $\left(\mathrm{h}^{2}=0,59\right.$ à 0,14 selon les espèces, cf. tableau 2), ce qui permet d'envisager une sélection efficace sur ce critère. Par ailleurs, une liaison génétique fortement négative entre le rythme de ventilation pulmonaire et la température rectale est rapportée dans un étude réalisées chez la vache $(-0,64)$ par Da Silva (1973). Dans la mesure où une bonne tolérance à la chaleur implique une faible température rectale et rythme respiratoire réduit, ce résultat suggère qu'il est difficile d'utiliser ce dernier critère dans une démarche de sélection.

\section{Autres indicateurs}

En réponse à un stress thermique aigu, la synthèse de protéines particulières appelées HSP (pour Heat Shock Protein) est stimulée dans le coeur, les muscles, les poumons et le cerveau chez de nombreuses espèces dont le porc. L'inhibition de la synthèse des HSP70 (HSP d'un poids moléculaire de $70 \mathrm{Kda}$ ) ou l'injection d'anti-corps anti-HSP70 augmente la sensibilité à la chaleur chez le rat (Moseley 1997), ce qui confirme leur implication dans la tolérance à la chaleur. Mais les modes d'action des HSP sont encore mal connus ; elles pourraient limiter la dénaturation des protéines, la désorganisation du cytosquelette et de la membrane cellulaire causée par une augmentation brusque de la température (Feder et Hofmann 1999). Chez le porc, des gènes codant pour les HSP ont été détectés sur les chromosomes 4,7 et 14 . Ces gènes semblent relativement indépendants du niveau de performances (Maak et al 1999), mais pourraient affecter certains paramètres de la qualité de la viande des porcs (Schwerin et al 1999).

\section{Conclusion}

La chaleur est l'un des principaux facteurs environnementaux affectant les performances de croissance et de reproduction du porc. Ses effets dépendent du stade physiologique de l'animal, de l'hygrométrie ambiante et, plus généralement, de la conduite d'élevage. En réponse à un stress thermique de longue durée, les porcs réagissent en diminuant ou déviant leur métabolisme énergétique et en augmentant les échanges de chaleur avec leur environnement. Pour atténuer les effets de la chaleur sur les performances des porcs, la solution la plus simple et la plus rapide consiste à modifier l'environnement thermique autour des animaux. D'autres solutions basées sur l'utilisation d'aliment à faible extra-chaleur peuvent également être appliquées. Cependant ces méthodes sont coûteuses et souvent mal adaptées aux conditions d'élevage tropicales, surtout lorsque l'on cherche à valoriser des matières premières locales, riches en fibres. Une alternative serait de pouvoir disposer d'animaux thermotolérants. Cette approche nécessite au préalable de comprendre les mécanismes physiologiques impliqués dans l'adaptation à la chaleur et la nature des antagonismes entre les caractères d'adaptation et de production. Cette première étape doit contribuer au choix des objectifs et des critères de sélection. Bien que peu de résultats soient disponibles dans la bibliographie concernant le porc, des indicateurs de la sensibilité à la chaleur, de la thermogenèse ou de la thermolyse pourraient être de bons critères à sélectionner pour l'obtention d'une lignée adaptée à la chaleur. 


\section{Références}

Abd-El-Gawad E.M., Khalifah M., Mérat P., 1992. Egg production of a dwarf (DW) and F1 cross between an experimental line and local lines in Egypt, especially in small scale production. In: 19th World's Poultry Congress, Amsterdam 2, 48-52.

Arthur P.F., Renand G., Krauss D., 2001. Genetic and phenotypic relationships among different measures of growth and feed efficiency in young Charolais bulls. Livest. Prod. Sci., 68, 131-139.

Baker R.L., Rege J.E.O., 1994. Genetic resistance to diseases and other stresses in improvement of ruminant livestock in the tropics. In: C. Smith (ed), Proceeding of the fifth Congress of Genetics Applied to Livestock Production, 20, 405-412. University of Guelph, Guelph, Ontario.

Beaumont C., Guillaumin S., Gearert P.A., MignonGrasteau S., Leclercq B., 1998. Genetic parameters of body weight of broiler chickens measured at $22^{\circ} \mathrm{C}$ or $32^{\circ} \mathrm{C}$. Br. Poult. Sci., 39, 488-491.

Berbigier P., 1975. Echanges thermiques au niveau de la peau des porcelets élevés en climat tropical. 1 . Influence des conditions climatiques et de la race. Ann. Zootech., 24, 423-432.

Bidanel J.P., Ricquet J., Chardon P., Hatey F., Le Roy P., Milan D., 2003. Apport des nouvelles biotechnologies aux programmes d'amélioration génétique du porc. Journées Recherche Porcine en France, 35, 355-368.

Black J.L., Mullan B.P., Lorschy M.L., Giles L.R., 1993. Lactation in the sow during heat stress. Livest. Prod. Sci., $35,153-170$.

Boissy A., Le Neindre P., Gastinel P.L., Bouix J., 2002. Génétique et adaptation comportementale chez les ruminants : persepectives pour améliorer le bien être en élevage. INRA Prod. Anim., 15, 373-382.

Bordas A., Minvielle F., 1997. Réponse à la chaleur de poules pondeuses issues de lignées sélectionnées pour une faible $(\mathrm{R}-)$ ou forte $(\mathrm{R}+)$ consommation alimentaire résiduelle. Genet. Sel. Evol., 29, 279-290.

Bordas A., Coquerelle G., Tixier-Boichard M., 1999. Evaluating local breeds and using major genes for farm chicken production in Taiwan. In: Symposium on Scientific cooperation in Agriculture between Institut National de la Recherche Agronomique (France) and Council of Agriculture (Taiwan, R.O.C.), Toulouse, France. INRACOA, 155-165.

Brown-Brandl T.M., Nienaber J.A., Yen J.T., 2000. Manual and thermal induced feed intake restriction on finishing barrows. I: effects on growth, carcass composition, and feeding behavior. Transactions of the ASAE, 43 (4), 987-992.

Burrow H.M., 2001. Variances and covariances between productive and adaptative traits and temperament in composite breed of tropical beef cattle. Livest. Prod. Sci., 70, 213-233.

Carvalho F.A., Lammoglia M.A., Simoes M.J., Randel R.D., 1995. Breed affects thermoregulation and epithelial morphology in imported and native cattle subjected to heat stress. J. Anim. Sci., 73, 3570-3573.

Collier R.J., Kobayashi Y., Gentry P., 2002. The use of genomics in genetic selection programs for environmental stress tolerance in domestic animals. In: 15th Conference on Biometeorology and Aerobiology -joint with 16th International Congress on Biometeorology of the International Society of Biometeorology (ISB), 28 October1 November 2002, Kansas City, MO, p 1-5.

Collin A., 2000. Effets d'une température ambiante élevée sur le métabolisme energétique du porcelet. Thèse ENSA Rennes, $163 \mathrm{p}$

Collin A., Lebreton Y., Fillaut M., Vincent A., Thomas F., Herpin P., 2001a. Effects of exposure to high temperature and feeding level on regional blood flow and oxidative capacity of tissues of piglets. Exp. Physiol., 86, 83-91.

Collin A., Van Milgen J., Le Dividich J., 2001b. Modelling the effect of high, constant temperature on food intake in young growing pigs. Anim. Sci. 72, 519-527.
D'Allaire S., Drolet R., Brodeur D., 1996. Sow mortality associated with high ambient temperatures. Can. Vet. J., 37 , 237-239.

Damon M., Vincent A., Lombardi A., Herpin P., 2000. First evidence of uncoupling protein-2 (UCP-2) and -3 (UCP-3) gene expression in piglet skeletal muscle and adipose tissue. Gene, 246, 133-141.

Da Silva R.G., 1973. Improving tropical beef cattle by simultaneous selection for weight and heat tolerance. Heritability and correlation of the traits. J. Anim. Sci., 37, 637-642.

Da Silva R.G., Arentes-Neto J.G., Helt-Filho S.V., 1988 Genetic aspects of the variation of the sweating rate and coat characteristics of Jersey Cattle. Rev. Bras.Genet., 11, 335-347.

Dauncey M.J., Ingram D.L., 1986. Acclimatization to warm or cold temperatures and the role of food intake. J. Theor. Biol., 11, 89-93.

De Basilio V., Picard M., 2002. La capacité de survie des poulets à un coup de chaleur est augmentée par une exposition précoce à une température élevée. INRA Prod. Anim., 15, 235-245.

De Lange C.F.M., Van Milgen J., Noblet J., Dubois S., Birkett S.H., 2002. Previous feeding level influences fasting heat production in growing pigs. J. Anim. Sci., 80 (suppl. 1), 63.

Delgado C., Rosegrant M., Steinfeld H., Ehui S., Courbois C., 1999. Livestock to 2020: The next food revolution Edition IFPRI, 2020 Discussion Papers on Food, Agriculture, and the Environment, n 28 , 99 p. IFPR (International Food Policy Research Institute), Washington DC (textes disponibles à http://www.ifpri.org/).

Enne G., Greppi G.F., 1993. Effect of temperature on sow performance. Pig News and Information, 14, 105N-112N.

Feder M.E., Hofmann G.E., 1999. Heat-shock proteins, molecular chaperones, and the stress response: evolutionary and ecological physiology. Annu. Rev. Physiol., 61 $243-282$

Ferrell C.L., Jenkins T.J., 1985. Cow type and the nutritional environment: nutrtional aspects. J. Anim. Sci., 61, 725-741.

Finch V.A., 1985. Comparison of non evaporative heat transfert in different cattle breeds. Aust. Aust J. Agric. Res. $36,497-508$.

Frisch J.E., 1981. Changes occuring in cattle as a consequence of selection for growth rate in stressful environment. J. Agric. Sci., 96, 23-38.

Gabarrou J.F., Geraert P.A., François N., Guillaumin S, Picard M., Bordas A., 1998. Energy balance of laying hens selected on residual food consumption. Br. Poult. Sci., 39, 79-89.

Geraert P.A., 1995. Déterminisme génétique de la resistance à la chaleur. In . 1ères Journées de la Recherche Avicole, Angers 1995. Ed. ITAVI Paris, 81-86.

Geuyen T.P.A., Verhagen J.M.F., Verstegen M.W.A., 1984 Effect of housing and temperature on metabolic rate of pregnant sows. Anim. Prod., 38, 477-485.

Giles L.R., Black J.L., 1991. Voluntary food intake in growing pigs at ambient temperatures above the zone of thermal comfort. In: E.S. Batterham (ed), Manipulating Pig Production III, 162-166. Australasian Pig Science Association, Institute of Animal Science Atwood.

Giles L.R., Bellinda D., Lowe R.F., 1998. Influence of diurnally fluctuating high temperature on growth and energy retention of growing pigs. Anim. Prod., 47, 467-474.

Guillouet Ph., Tribout T., Bussière J.F., Bertaud G., Bidanel J.P., Terqui M., 1999. Analyse des facteurs de variation de la production spermatique de verrats d'insémination artificielle. Journées de la Recherche Porcine en France, 31, 45-52.

Hammond A.C., Chase C.C., Bowers E.J., Olson T.A. Randel R.D., 1998. Heat tolerance in Tuli-, Senepol-, and Brahman-sired F1 Angus Heifers in Floridia. J. Anim. Sci. $76,1568-1577$. 
Holmes C.W., Close W.H., 1977. The influence of climatic variables on energy metabolism and associated aspects of productivity in the pig. In: Haresign W., Swan H. and Lewis D. (eds), Nutrition and the climatic environment (4), 51-73. Butterworths, Studies in the agricultural and food sciences, London.

Holmes C.W., McLean N.R., 1974. The effect of low temperatures on the energy metabolism of sows. Anim. Prod., $19,1-12$.

Hotovy S.K., Johnson K.A., Johnson D.E., Carstens G.E., Bourdon R.M., Seidel G.E., 1991. Variation among twin beef cattle in maintenance energy requirements. J. Anim. Sci. 69, 940-946.

Ingram D.L., 1973. Heat loss and its control in pigs. In: Monteith J.L. and Mount L.E. (eds), Heat loss from animals and man. Assessment and Control, 235-254. Butterworths, London.

Jenkinson D., Mason I.L., Nay T., 1975. Inheritance of some sweat gland and hair follicle characteristics in cattle. Aust. J. Biol. Sci., 28, 417-425.

Johnson Z.B., Chewning J.J., Nugent R.A., 1999. Genetic parameters for production traits and measures of residual feed intake in Large White swine. J. Anim. Sci., 77, 16791685 .

Kabuga J.D., Annor S.Y., 1991. Seasonal influence on the reproductive performance of swine in the humid zone of Ghana. Intl J. Biometeorology, 35, 208-213.

Kadzere C.T., Murphy M.R., Silznikove N., Maltz E., 2002. Heat stress in lactating dairy cows: a review. Livest. Prod. Sci., 77, 59-91.

Kemp B., Verstegen M.W.A., Verhagen J.M.F, van der Hel W., 1987. The effect of environmental temperature and feeding level on energy and protein retention of individual housed pregnant sows. Br. J. Nutr., 44, 275-283.

Kemp B., Verstegen M.W.A., den Hartog L.A., Grooten H.J.G., 1989. The effect of environmental temperature on metabolic rate and partitoning of energy intake in breeding boars. Livest. Prod. Sci., 23, 329-340.

Labroue F., Maignel L., Sellier P., Noblet J., 1999. Consommation résiduelle chez le porc en croissance alimenté à volonté : méthode de calcul et variabilité génétique. Journées de la Recherche Porcine en France, 31, 167-174.

Lagarrigue S, Daval S., Bordas A, Douaire M, 2000. Hepatic lipogenesis gene expression in two experimental egg-laying lines divergently selected on residual feed consumption. Genet. Sel. Evol., 32, 205-216.

Le Bellego L., Noblet J., Van Milgen J., 2002a. Effect of high temperature and low protein diets on performance of growing-finishing pigs. J. Anim. Sci., 80, 691-701.

Le Bellego L, Van Milgen J, Noblet J, 2002b. Effects of high ambient temperature on protein and lipid deposition and energy utilization in growing pigs. Anim. Sci., 75, 85-96.

Le Dividich J., Rinaldo D., 1989. Effets de l'environement thermique sur les performances du porc en croissance. Journées de la Recherche Porcine en France, 21, 219-230.

Maak S., Hardge T., Wimmers K., von Lengerken G. Leuthold G., 1999. No association between mutations in the porcine HSP 70.2 gene promoter and performance traits in an experimental F-2 population. Archiv. Tierzucht, 42, 141 143.

Mackinnon M.J., Meyer K., Hetzel D.J.S., 1991. Genetic variation and covariation for growth, parasite resistance and heat tolerance in tropical cattle. Livest. Prod. Sci., 27, 105-122.

Maloyan A., Palmon A., Horowitz M., 1999. Heat acclimation increases the basal HSP72 level and alters its production dynamics during heat stress. Amer. J. Physiol., 76, R1506-R1515.

Mc Glone J.J., Stansbury W.F., Tribble L.F., 1988. Management of lactating sows during heat stress: effects of water drip, snout coolers, floor type and a high energy-density diet. J. Anim. Sci., 66, 885-891.
Mérat P., 1986. Potential usefulness of the NA (naked neck)gen in poultry production. World Poult. Sci. J., 42 124-142.

Moody D.E., Pomp D., Nielsen M.K., Van Vleck L.D. 1999. Identification of quatitative trait loci influencing traits related to energy balance in selection and inbred lines of mice. Genetics, 152, 699-711.

Morris C.A., Jones K.R., Wilson J.A., 1989. Heritability of rectal temperature and relationships with growth in young cattle in a temperate climate. NZ J. Agric. Res., 32, 375-378.

Moseley P.L., 1997. Heat shock proteins and heat adaptation of the whole organism. J. Appl. Physiol., 83, 1413 1417.

Mousel M.R., Stroup W.W., Nielsen M.K., 2001 Locomotor activity, core body temperature, and circadian rythms in mice selected for high and low heat loss. J. Anim. Sci., $79,861-868$

Mrode R.A., Kennedy B.W., 1993. Genetic variation in measures of food efficiency in pigs and their genetic relationships with growth rate and backfat. Anim. Prod., 56 225-232.

Nielsen M.K., Freking B.A., Jones L.D., Nelson S.M. Vorderstrasse T.L., Hussey B.A., 1997a. Divergent selection for heat loss in mice: II. Correlated responses in feed intake, body mass, body composition, and number born through fifteen generations. J. Anim. Sci., 75, 1469-1476.

Nielsen M.K., Jones L.D., Freking B.A., De Shazer J.A. 1997b. Divergent selection for heat loss in mice: I. Selection applied and direct response through fifteen generations. J. Anim. Sci., 75, 1461-1468.

Nienaber J.A., Hahn G.L., Eigenberg R.A., Korthals R.L., Yen J.T., Harris D.L., 1997. Genetic and heat stress interaction effects on finishing swine. In : Bottcher R.W. and Hof S.J. (eds), Livestock Environment, 2, 1017-1023. American Society of Agricultural Engineers, Bloomington, Minnesota.

Nienaber J.A., Hahn G.L., Yen J.T., 1987. Thermal environment effects of growing-finishing swine. I. Growth, feed intake and heat production. Transactions of the American Society of Agricultural Engineers, 30, 1772-1775.

Noblet J., Etienne M., 1987. Body composition, metabolic rate and utilization of milk nutrients in suckling piglets. Reprod. Nutr. Dev., 27, 829-839.

Noblet J., Dourmad J.Y., Le Dividich J., Dubois S., 1989. Effect of ambient temperature and addition of straw or alfalfa in the diet on energy metabolism in pregnant sows. Livest. Prod. Sci., 21, 309-324.

Noblet J., Dourmad J.Y., Etienne M., Le Dividich J., 1997. Energy metabolism in pregnant sows and newborn pigs. J. Anim. Sci., 75, 2708-2714.

Noblet J., Karege C., Dubois S., Van Milgen J., 1999. Metabolic utilization of energy and maintenance requirements in growing pigs: effects of sex and genotype. J. Anim Sci., 77, 1208-1216.

Obeidah A., Mostageer A., Shafie M.M., 1974. Genetic and phenotypic parameters of body temperature and respiration rate in Fayoumi chiks. Ann. Génét. Sél. Anim., 6, 267 274

Olson T.A., Hammond A.C., Chase Jr., 2003. Evidence of a major gene influencing hair length and heat tolerance in Bos Taurus cattle. J. Anim. Sci., 81, 80-90.

Peltoniemi O.A.T., Tast A., Love R.J., 2000. Factors effecting reproduction in the pig: seasonal effects and restricted feeding of the pregnant gilt and sow. Anim. Reprod. Sci., 60 $61,173-184$.

Prunier A., Quesnel H., Messias de Bragança M. Kermabon A.Y., 1996. Environmental and seasonal influences on the return-to-oestrus after weaning in primiparous sows: a review. Livest. Prod. Sci., 45, 103-110.

Quesnel H., Prunier A., 1995. L'ovulation après le tarissement des truies : mécanismes physiologiques et facteurs de variation. INRA Prod. Anim., 8, 165-176. 
Quiniou N., Noblet J., 1999. Influence of high ambient temperatures on performance of multiparous lactating sows. J. Anim. Sci., 77, 2124-2134.

Quiniou N., Dubois S., Noblet J., 2000a. Voluntary feed intake and feeding behaviour of group-housed growing pigs are affected by ambient temperature and body weight. Livest. Prod. Sci., 63, 245-253.

Quiniou N., Renaudeau D., Collin A., Noblet J., 2000b. Effets de l'exposition au chaud sur les caractéristiques de la prise alimentaire du porc à différents stades phydiologiques. INRA Prod. Anim., 13, 233-245.

Quiniou N., Gaudré D., Guillou D., 2001. Influence de la température ambiante et de la concentration en nutriments de l'aliment sur les performances de lactation des truies selon le rang de portée. Journées de la Recherche Porcine en France, 33, 157-163.

Raimbault S., Dridi S., Denjean F., Lachuer J., Couplan E., Bouillaud F., Bordas A., Duchamp C., Taouis M., Riquier D., 2001. An uncoupling protein homologue putatively involved in facultative muscle thermogenesis in birds. Biochem. J., 353, 441-444.

Renaudeau D., 2001. Adaptation nutritionnelle et physiologique aux températures ambiantes élevées chez la truie en lactation. Thèse Université de Rennes 1,160 p.

Renaudeau D., Quiniou N., Noblet J., 2001. Effects of exposure to high ambient temperature and dietary protein level on performance of multiparous lactating sows. J. Anim. Sci., 79, 1240-1249.

Renaudeau D., Anaïs C., Noblet J., 2003a. Effects of dietary fiber on performance of multiparous lactating sows in a tropical climate. J. Anim. Sci., 81, 717-725.

Renaudeau D., Noblet J., Dourmad J.Y., 2003b. Effect of ambient temperature on mammary gland metabolism in lactating sows. J. Anim. Sci., 81, 217-231.

Ricquier D., Bouillaud F., 2000. The uncoupling protein homologues : UCP1, UCP2, UCP3, stUCP and atUCP. Biochem. J., 345, 161-179.

Rinaldo D., Le Dividich J., 1991. Influence de la température ambiante sur les performances de croissance du porc. INRA Prod. Anim., 4, 57-65.

Rinaldo D., Le Dividich J., Noblet J., 2000. Adverse effects of tropical climate on voluntary feed intake and performance of growing pigs. Livest. Prod. Sci., 66, 223-234.

Sartor V., Baeta F.C., Ferreira A.S., Cecon P.R., Tinoco I.F.F., 1999. Effect of the systems of evaporative cooling in the performance of lactation sows and their litters. [Portuguese]. Engenharia Agricola, 18, 4-13.

Schoenherr W.D., Stahly T.S., Cromwell G.L., 1989. The effects of dietary fat or fiber addition on yield and composition of milk from sows housed in a warm or hot environment. J. Anim. Sci., 67, 482-495.

Schwerin M., Hagendorf A., Furbass R., Teuscher F., 1999. The inducible stress protein 70.2 gene - a candidate gene for stress susceptibility in swine. Archiv. für Tierzucht - Archives of animal breeding, 42, 61-66.
Settar P., Yalçin S., Türkmut L., Özkan S., Cahanar A. 1999. Season by genotype interaction related to broiler growth rate and heat tolerance. Poult. Sci., 78, 1353-1358.

St-Pierre N.R., Cobanov B., Schnitkey G., 2003. Economic losses from heat stress by US livestock industries. J. Dairy Sci., 86(E.Suppl.), E52-E77.

Stahly T.S., Cromwell G.L., 1979. Effect of environmental temperature and dietary fat supplementation on the performance and carcass characteristics of growing and finishing swine. J. Anim. Sci., 49, 1478-1488.

Steinbach J., 1976. Reproductive performance in high producing pigs under tropical conditions. World Anim. Rev., 19, 43-47.

Taouis M., De Basilio V., Mignon-Grasteau S, Crochet S. Bouchot C., Bigot K., Collin A., Picard M., 2002. Early age thermal conditionning reduces uncoupling protein messenger RNA expression in pectoral muscle of broiler chicks at seven days of age. Poult. Sci., 80, 1640-1643.

Tauson A.H., Chwalibog A., Ludviqsen J., Jakobsen K. Thorbek G., 1998. Effect of short-term exposure to high ambient temperatures on gas exchange and heat production in boars of different breeds. Anim. Sci., 66, 431-440.

Turner H.G., 1982. Genetic varaiation of rectal temperature in cows and its relationship to fertility. Anim. Prod., 35, 401-412.

Turner H.G., 1984. Variation in rectal temperature of cattle in a tropical environment and its relation to growth rate. Anim. Prod., 38, 417-427.

Van Milgen J., Noblet J., Dubois S., Bernier J.F., 1997. Dynamic aspects of oxygen consumption and carbon dioxide production in swine. Br. J. Nutr., 78, 397-410.

Van Milgen J., Bernier J.F., Le Cozler Y., Dubois S., Noblet J., 1998. Major determinants of fasting heat production and energetic cost of activity in growing pigs of different body weight and breed/castration combination. Br. J. Nutr., 79, 509-517.

Van Milgen J., Noblet J., Dubois S., 2000. Modélisation des composantes de la dépense énergétique chez le porc. Journées de la Recherche Porcine en France, 32, 235-240.

Van Milgen J., Noblet J., Dubois S., 2001. Energetic efficiency of starch, protein and lipid utilization in growing pigs. J. Nutr., 131, 1309-1318.

Verstegen M.W.A., Curtis S.E., 1988. Energetics of sows and gilts in gestation crates in the cold. J. Anim. Sci., 66, 2865-2875.

Verstegen M.W.A., Brascamp E.W., van der Hel W., 1978. Growing and fattening of pigs in relation to temperature of housing and feeding level. Can. J. Anim. Sci., 58, 1-13.

Wettmann R.P., Bazer F.W., 1985. Influence of environmental temperature on prolificacy of pigs. J. Reprod. Fert. 33(suppl.), 199-208.

Wettmann R.P., Wells M.E., Johnson R.K., 1979 Reproductive characteristics of boars during and after exposure to increased ambient temperature. J. Anim. Sci. 49, 1501-1505.

Ye W., Xin H., 2000. Thermographical quantification of physiological and behavioral responses of group-housed young pigs. Transactions of the American Society of Agricultural Engineers, 43(6), 1843-1851. 


\section{Abstract}

Attenuate the effects of high ambient temperature on pig performance: the genetic selection.

Ambient temperature is one of the most important climatic factors affecting pig performances in the summertime in temperate areas and throughout the year in tropical areas. Exposure to high ambient temperature directly affects male and female reproduction function and indirectly affects lactation and growth performance via a reduced voluntary feed intake. After a short presentation of the mechanisms implied in thermoregulation and the effects of high ambient temperature on pig performance according to the physiological state, this review focuses on the methods that could be used to reduce the negative effect of heat stress and it also highlights on the possibilities for genetic improvement of heat tolerance. To establish whether the selection of pigs for heat stress is feasible, the physiological mecha- nisms implied in heat adaptation processes and the relationship between adaptation and production traits must be better understood. This first step is necessary to choose the best selection criteria and breeding program for the genetic improvement of heat tolerance. In contrast to other species (ruminant and chickens), genetic diversity for heat tolerance within or between breed and potential selection criteria are little known in the pig. However, some indicators of heat sensitivity (Heat Shock Protein), heat production (residual consumption, Uncoupling protein) or heat dissipation (respiratory rate, cutaneous heat conductivity) could be used as the selection criteria.

RENAUDEAU D., MANDONNET N., TIXIERBOICHARD M., NOBLET J., BIDANEL J-P., 2004 Atténuer les effets de la chaleur sur les performances des porcs : la voie génétique. INRA Prod. Anim., 17, 93-108. 